Proyecciones

Vol. 27, No 3, pp. 307-318, December 2008.

Universidad Católica del Norte

Antofagasta - Chile

\title{
ABOUT AN EXISTENCE THEOREM OF THE HENSTOCK - FOURIER TRANSFORM
}

\author{
FRANCISCO JAVIER MENDOZA TORRES \\ BENEMÉRITA UNIVERSIDAD AUTÓNOMA DE PUEBLA, MÉXICO \\ JUAN ALBERTO ESCAMILLA REYNA \\ BENEMÉRITA UNIVERSIDAD AUTÓNOMA DE PUEBLA, MÉXICO \\ and \\ MA. GUADALUPE RAGGI CÁRDENAS \\ BENEMÉRITA UNIVERSIDAD AUTÓNOMA DE PUEBLA, MÉXICO \\ Received : June 2008. Accepted : November 2008 \\ Dedicated to the memory of $V$. A. Borovikov
}

\begin{abstract}
We show that if $f$ is lying on the intersection of the space of Henstock-Kurzweil integrable functions and the space of the bounded variation functions in the neighborhood of $\pm \infty$, then its Fourier Transform exists in all $\mathbf{R}$. This result is more general than the classical result which enunciates that if $f$ is Lebesgue integrable, then the Fourier Transform of $f$ exists in all $\mathbf{R}$, because we also have proved that there are functions which belong to the intersection of the space of the Henstock-Kurzweil integrable functions and the space of the bounded variation functions which are not Lebesgue integrable.
\end{abstract}

Key words: Henstock-Kurzweil Integral, Bounded Variation Function, Lebesgue Integral.

2000 Mathematics Subjet Classification. 26A39, 26A45, $26 A 42$. 


\section{Introduction}

In [6] (which explains Henstock-Kurzweil Fourier transforms), Erik Talvila shows several results about Fourier Transform in $H K(\mathbf{R})$, the space of the Henstock-Kurzweil integrable functions over $\mathbf{R}$. A particular result which has captured our attention is the proposition 2 (b) which talks about the existence of Fourier Transform in the $H K_{l o c}(\mathbf{R})$ space, which is enunciated as follows

If $f$ is locally $H K$-integrable and of bounded variation in a neighborhood of infinity with limit zero at infinity, then, $\widehat{f}$ exists in $\mathbf{R}$.

It is obvious that under these conditions, it is not necessarily true that the Fourier Transform exists in zero (for instance). The hypothesis proposed by Talvila leads to the idea that if we impose a condition where $f \in H K(\mathbf{R}) \cap B V([ \pm \infty])$, here $B V([ \pm \infty])$ is the space of the functions which are of bounded variation in some neighborhood of $\pm \infty$, then $\widehat{f}(s)$ exists for all $s \in \mathbf{R}$. A problem which might arise is that $H K(\mathbf{R}) \cap B V([ \pm \infty]) \subseteq L(\mathbf{R})$, where $L(\mathbf{R})$ is the Lebesgue integrablefunctions space, for which the existence of the Fourier Transform, for all $s \in \mathbf{R}$, in $H K(\mathbf{R}) \cap B V([ \pm \infty])$ could not represent a progress. Just this situation, we have developed two points in this paper:

1 Contention relations of the space $H K(I) \cap B V(I)$ for unbounded intervals.

2 An existence theorem of $\widehat{f}(s)$ in $H K(\mathbf{R}) \cap B V([ \pm \infty])$.

With respect to the point $\mathbf{1}$, we know that if $I$ is a compact interval, every bounded variation function is Lebesgue integrable. Because every Lebesgue integrable function is $H K$-integrable, we will have that, for bounded intervals:

$$
B V(I) \subset L(I) \subset H K(I),
$$

for which $H K(I) \cap B V(I) \subset L(I)$, and this contention is proper. For instance, the function $f:[0,1] \rightarrow \mathbf{R}$ defined as

$$
f(x)=\left\{\begin{array}{cc}
x \sin (1 / x), & \text { si } x \in(0,1] \\
0, & \text { si } x=0
\end{array}\right.
$$

is in $L(I)-B V(I)$.

If $I$ is an unbounded interval of the shape $[a, \infty]$ or $[-\infty, a]$, then it is a natural question to ask ourselves if the contention relations of the spaces 
$B V(I), L(I)$, and $H K(I)$ are the same as when $I$ is compact. To answer to this, we show a family of functions which is useful to us for showing that the former contentions behave in a different way. Among these relations, the more significative is:

$$
H K(I) \cap B V(I) \nsubseteq L(I) .
$$

With respect to the point $\mathbf{2}$, we will prove that:

If $f \in H K(\mathbf{R}) \cap B V([ \pm \infty])$, then $\widehat{f}(s)$ exists for all $s \in \mathbf{R}$.

\section{The Spaces $H K(I)$ and $B V(I)$}

\subsection{The Henstock-Kurzweil Integral}

For compact intervals in $\mathbf{R}$, the Henstock-Kurzweil integral is defined in the following way:

Definition 1.1. Let $f:[a, b] \rightarrow \mathbf{R}$ be a function, we will say that $f$ is Henstock-Kurzweil integrable if there exists $A \in \mathbf{R}$, which satisfies the following:

for each $\varepsilon>0$ exists a function $\gamma_{\varepsilon}:[a, b] \rightarrow(0, \infty)$ such that for every partition labeled $P=\left\{\left(\left[x_{i-1}, x_{i}\right], t_{i}\right)\right\}_{i=1}^{n}$, where $t_{i} \in\left[x_{i-1}, x_{i}\right]$, if

$$
\left[x_{i-1}, x_{i}\right] \subset\left[t_{i}-\gamma_{\varepsilon}\left(t_{i}\right), t_{i}+\gamma_{\varepsilon}\left(t_{i}\right)\right] \quad \text { for } i=1,2, \ldots, n,
$$

then

$$
\left|\sum_{i=1}^{n} f\left(t_{i}\right)\left(x_{i}-x_{i-1}\right)-A\right|<\varepsilon .
$$

The function $\gamma_{\varepsilon}$ is commonly called gauge, and if the partition $P$ complies with the condition (1.1), we will say that it is $\gamma_{\varepsilon}$-fine. The number $A$ is called as the integral of $f$ over $[a, b]$ and it is denoted as

$$
A=\int_{a}^{b} f
$$

If $f$ is defined over an interval $[a, \infty]$, we condition it to $f(\infty)=0$. In this case, given a gauge function $\gamma_{\varepsilon}:[a, \infty] \rightarrow(0, \infty)$, where $\gamma_{\varepsilon}(\infty) \in \mathbf{R}^{+}$, we will say that the labeled partition $P=\left\{\left(\left[x_{i-1}, x_{i}\right], t_{i}\right)\right\}_{i=1}^{n+1}$ is $\gamma_{\varepsilon}$-fine if:

a) $x_{0}=a, x_{n+1}=\infty$.

b) $\left[x_{i-1}, x_{i}\right] \subset\left[t_{i}-\gamma_{\varepsilon}\left(t_{i}\right), t_{i}+\gamma_{\varepsilon}\left(t_{i}\right)\right]$, for $i=1,2, \ldots, n$ 
c) $\left[x_{n}, \infty\right] \subset\left[1 / \gamma_{\varepsilon}(\infty), \infty\right]$.

Thus, the function will be integrable if it satisfies definition 1.1, and also the condition of that the partition $P$ be $\gamma_{\varepsilon}$-fine acording to the previous incises. In addition, these conditions cause that the last term of $\sum_{i=1}^{n+1} f\left(t_{i}\right)\left(x_{i}-x_{i-1}\right)$ is zero and thus this sum is finite. For functions defined over intervals $[-\infty, a]$ or $[-\infty,+\infty]$ we do similar considerations.

Through the theory of this integral we have that $f:[-\infty, \infty] \rightarrow \mathbf{R}$ is an integrable function, if and only if, $f$ is an integrable function over the intervals $[a, \infty]$ y $[-\infty, a]$. In this case

$$
\int_{-\infty}^{\infty} f=\int_{-\infty}^{a} f+\int_{a}^{\infty} f
$$

We denote as

$H K(I)=\{f: I \rightarrow \mathbf{R} \mid f$ is integrable in the sense of Henstock-Kurzweil in $I\}$

Some features of $H K(I)$ are the following:

1. It is a vector space, i.e.: the sum of functions and the product by scalars of Henstock-Kurzweil integrable functions are integrable. The integral is a linear functional over this space.

2. It contains the Riemann and Lebesgue integrable functions. Also, the functions whose Riemann or Lebesgue improper integrals exist, and its values coincide.

3. It generalizes the Fundamental Theorem of Calculus, in the sense that every derivative function is integrable. This does not happen with Riemann and Lebesgue integrals. In this case we have:

$$
\int_{a}^{b} f^{\prime}=f(b)-f(a)
$$

4. Since we know, in Riemann's integral, if two functions are integrable, then their product is also integrable. In the case of the integral of $H K$, this is not true. Nevertheless, the product of a $H K$-integrable function by a bounded variation function, is in fact integrable.

5. The $H K$ integral is not an absolute integral. This asseveration is in the sense that if $f$ is $H K$-integrable, it does not imply that $|f|$ is so. When $|f|$ and $f$ are integrable, we say that $f$ is absolutely $H K$-integrable. 
6. The space of the absolutely $H K$-integrable functions is $L(I)$, the space of the Lebesgue-integrable functions.

The following theorems plays an important roll into this theory of integration, and also in the demonstration of our results. The intervals we have considered could be compact or unbounded.

Theorem 1.1. Let $f \in H K(I)$ be a function. Then $|f|$ is integrable, if and only if, $F(x)=\left[\int_{a}^{x} f\right] \in B V(I)$. In this case we have that the total variation of $F$ in $I$ is:

$$
V(F, I)=\int_{I}|f|
$$

Theorem 1.2. If $f, g \in H K(I)$ and $|f(x)| \leq g(x)$ for all $x \in I$, then $f \in L(I)$. In addition,

$$
\left|\int_{I} f\right| \leq \int_{I}|f| \leq \int_{I} g
$$

Theorem 1.3 (Hake's). Let $I=[a, \infty]$ and $f: I \rightarrow R$ be a function, then $f \in H K(I)$, if and only if, $f \in H K([a, c])$ for any compact interval $[a, c] \subset[a, \infty)$, and there exists $A \in \mathbf{R}$ such that

$$
\lim _{c \rightarrow \infty} \int_{a}^{c} f=A
$$

In this case we have that: $A=\int_{a}^{\infty} f$.

This last theorem is also valid for intervals $[-\infty, b]$ or $[-\infty, \infty]$, with its corresponding adaptation.

Theorem 1.4 (Chartier-Dirichlet). Let $f, \varphi:[a, \infty] \rightarrow \mathbf{R}$ be functions, and suppose that:

a) $f \in H K([a, c])$ for all $c \geq a$, and $F(x)=\int_{a}^{x} f$ is bounded in $[a, \infty)$.

b) $\varphi$ is monotone and $\lim _{x \rightarrow \infty} \varphi(x)=0$.

Then $f \varphi \in H K([a, \infty])$. 


\subsection{Bounded Variation Functions in Unbounded Intervals}

We suppose that the reader knows the concept of bounded variation for functions defined in bounded intervals. We will denote the total Variation of $f$ in $I$ as $V(f, I)$. For unbounded intervals, the definition of the concept about bounded variation is:

Definition 1.2. Let $f:[a, \infty] \rightarrow \mathbf{R}$ be a function. We say that $f$ is of bounded variation in $I=[a, \infty]$ if there exists $M>0$ in such a way that, for all $b \geq a$, it is fulfilled:

$$
V(f,[a, b]) \leq M
$$

The total variation of $f$ in $I$ will be:

$$
V(f, I)=\sup _{a \leq b} V(f,[a, b]) .
$$

For $I=[-\infty, b]$ the definition is analogous. If $I=[-\infty, \infty]$, the total variation is defined as

$$
V(f, I)=\sup _{a \leq b} V(f,[a, b])
$$

See that in (1.3) and in (1.4), we have, respectively, that:

$$
V(f,[a, \infty])=\lim _{b \rightarrow \infty} V(f,[a, b])
$$

and

$$
V(f,[-\infty, \infty])=\lim _{\substack{a \rightarrow-\infty \\ b \rightarrow \infty}} V(f,[a, b]) .
$$

Let $B V(I)=\{f: I \rightarrow \mathbf{R} \mid f$ is of bounded variation in $I\}$ be, where $I$ is an unbounded interval. Some features belonging to $B V(I)$ are the following:

a) $B V(I)$ is a vector space.

b) $f \in B V(I)$, if and only if, there exist $f_{1}$ and $f_{2}$ which are increasing bounded-functions such that $f=f_{1}-f_{2}$.

c) If $I=[a, \infty]$, then $\lim _{x \rightarrow \infty} f(x)$ exists. For cases $[-\infty, b],[-\infty . \infty]$ we have similar results. 


\section{Contention relations of the space $H K(I) \cap B V(I)$, for un- bounded intervals}

In the same way as commented in the introductory section, if $I$ is a compact interval, then

$$
B V(I) \subset L(I) \subset H K(I),
$$

which means that: $H K(I) \cap B V(I) \underset{\subsetneq}{\subset} L(I)$.

Now, if $I$ is unbounded, the first two observations which we can see are

$$
B V(I) \nsubseteq L(I)
$$

and

$$
L(I) \nsubseteq H K(I) \cap B V(I) .
$$

It is easy to demonstrate that the function $f(x)=1 / x$ defined in $[1, \infty]$, is of bounded variation, and

$$
V(f,[1, \infty])=1
$$

and

$$
\int_{1}^{\infty} \frac{1}{x}=\infty
$$

This implies that (2.1) is true.

For (2.2), we consider the function $f:[0, \infty] \rightarrow \mathbf{R}$

$$
f(x)=\left\{\begin{array}{cc}
\sqrt{x} \sin (1 / x), & \text { si } x \in(0,1] \\
0, & \text { si } x=0, x \in(1, \infty],
\end{array}\right.
$$

which is in $L([0, \infty])-B V([0, \infty])$.

Next, we will prove that: $H K(I) \cap B V(I) \nsubseteq L(I)$.

Theorem 2.1. Let $\varphi:[a, \infty] \rightarrow R$ be a non-negative function, which is decreasing to zero when $x \rightarrow \infty$. If $\varphi \notin H K[a, \infty])$, then the functions: $\varphi(t) \sin (t)$ y $\varphi(t) \cos (t)$ are in $H K([a, \infty])-L([a, \infty])$. 
Proof. Prove that $\varphi(t) \sin (t) \notin L([a, \infty])$. The demonstration that $\varphi(t) \cos (t) \notin L([a, \infty])$ is done in a similar way.

Suppose that $n_{0}$ is the natural minor number for which $a<\left(1+4 n_{0}\right) \pi / 4$.

For $x \in[a, \infty]$ we have

$$
\begin{gathered}
|\sin x| \geq \frac{1}{\sqrt{2}} \text { if and only if } \\
x \in \cup_{n=n_{0}}^{\infty}\left[(1+4 n) \frac{\pi}{4},(3+4 n) \frac{\pi}{4}\right] .
\end{gathered}
$$

Given $n \in \mathbf{N}$, as $(3+4 n) \pi / 4<(1+n) \pi$, we have that:

$$
\begin{aligned}
\int_{a}^{(1+n) \pi} \varphi(t)|\sin x| & \geq \frac{1}{\sqrt{2}} \sum_{i=n_{0}}^{n} \int_{(1+4 i) \pi / 4}^{(3+4 i) \pi / 4} \varphi(t) \\
& \geq \frac{1}{\sqrt{2}} \sum_{i=n_{0}}^{n} \int_{(1+4 i) \pi / 4}^{(3+4 i) \pi / 4} \varphi((3+4 i) \pi / 4) \\
& =\frac{\pi}{2 \sqrt{2}} \sum_{i=n_{0}}^{n} \varphi((3+4 i) \pi / 4) \\
& \geq \frac{\pi}{2 \sqrt{2}} \sum_{i=n_{0}}^{n} \varphi((1+i) \pi)
\end{aligned}
$$

On the other hand, we have that:

$$
\begin{aligned}
\int_{a}^{(1+n) \pi} \varphi(t) & =\int_{a}^{n_{0} \pi} \varphi(t)+\int_{n_{0} \pi}^{(1+n) \pi} \varphi(t) \\
& =\int_{a}^{n_{0} \pi} \varphi(t)+\sum_{i=n_{0}}^{n} \int_{i \pi}^{(1+i) \pi} \varphi(t) \\
& \leq \int_{a}^{n_{0} \pi} \varphi(t)+\pi \sum_{i=n_{0}}^{n} \varphi(i \pi) .
\end{aligned}
$$

Because $\varphi \notin H K([a, \infty])$, then $\int_{a}^{\infty} \varphi(t)=\infty$ and from (2.5)

$$
\sum_{i=n_{0}}^{\infty} \varphi(n \pi)=\infty
$$

Using (2.6) and approaching $n \rightarrow \infty$ in (2.4) we conclude that $\varphi(t) \sin (t) \notin L([a, \infty])$.

Now, because for any $x \in[a, \infty)$ 


$$
|F(x)|=\left|\int_{a}^{x} \sin (t)\right| \leq 2 \text { y }|G(x)|=\left|\int_{a}^{x} \cos (t)\right| \leq 2,
$$

then by the theorem 1.4. (from Chartier-Dirichlet) we have that: $\varphi(t) \sin (t) \varphi(t)$ y $\varphi(t) \cos (t)$ are in $H K[a, \infty]$.

Corollary 2.1. For any $a>0$, the function

$f(t)=\sin (t) / t \in H K([a, \infty])-L([a, \infty])$.

Corollary 2.2. Let $1>\alpha>0$. The function $f_{\alpha}:\left[\pi^{1 / \alpha}, \infty\right] \rightarrow \mathbf{R}$ defined as $f_{\alpha}(t)=\frac{\sin \left(t^{\alpha}\right)}{t}$ is in $H K\left[\pi^{1 / \alpha}, \infty\right]-L\left(\left[\pi^{1 / \alpha}, \infty\right]\right)$.

Proof. Let $c>\pi^{1 / \alpha}$. Doing a change of variable $u=t^{\alpha}$ we have that

$$
\int_{\pi^{1 / \alpha}}^{c} \frac{\sin \left(t^{\alpha}\right)}{t} d t=\frac{1}{\alpha} \int_{\pi}^{c^{\alpha}} \frac{\sin (u)}{u} d u .
$$

Because $\sin (u) / u \in H K[\pi, \infty]-L([\pi, \infty])$, we have that:

$$
\lim _{c \rightarrow \infty} \int_{\pi^{1 / \alpha}}^{c} \frac{\sin \left(t^{\alpha}\right)}{t} d t \text { exists }
$$

i.e., $f_{\alpha} \in H K\left[\pi^{1 / \alpha}, \infty\right]-L\left(\left[\pi^{1 / \alpha}, \infty\right]\right)$.

Theorem 2.2. Let $1>\alpha>0$. The function $f_{\alpha}$ defined in corollary $\mathbf{2 . 2}$ belongs to $B V\left(\left[\pi^{1 / \alpha}, \infty\right]\right)$.

Proof. Let $x \in\left(\pi^{1 / \alpha}, \infty\right)$. We know that $f_{\alpha}^{\prime} \in H K\left(\left[\pi^{1 / \alpha}, x\right]\right)$. Because

$$
f_{\alpha}^{\prime}(t)=\frac{\alpha \cos \left(t^{\alpha}\right)}{t^{2-\alpha}}-\frac{\sin \left(t^{\alpha}\right)}{t^{2}}
$$

we have that

$$
\left|f_{\alpha}^{\prime}(t)\right| \leq \frac{\alpha}{t^{2-\alpha}}+\frac{1}{t^{2}}
$$

The function $g(t)=\frac{\alpha}{t^{2-\alpha}}+\frac{1}{t^{2}} \in H K\left(\left[\pi^{1 / \alpha}, x\right]\right)$, then by (2.8) and the Theorem 1.2. we conclude that: $f_{\alpha}^{\prime} \in L\left(\left[\pi^{1 / \alpha}, x\right]\right)$ and 


$$
\begin{aligned}
& \int_{\pi^{1 / \alpha}}^{x}\left|f_{\alpha}^{\prime}\right| \leq \int_{\pi^{1 / \alpha}}^{x}\left(\frac{\alpha}{t^{2-\alpha}}+\frac{1}{t^{2}}\right) d t \\
= & \left(\frac{1}{\alpha-1}\right)\left[x^{\alpha-1}-\pi^{\frac{\alpha-1}{\alpha}}\right]-\frac{1}{x}+\frac{1}{\pi^{1 / \alpha}} .
\end{aligned}
$$

Considering that $1-\alpha>0$, then using Theorem 1.1. and (2.9) we have that

$$
V\left(f,\left[\pi^{1 / \alpha}, x\right]\right) \leq \frac{1}{(1-\alpha) \pi^{(1-\alpha) / \alpha}}+\frac{1}{\pi^{1 / \alpha}} .
$$

Therefore, $f_{\alpha} \in B V\left(\left[\pi^{1 / \alpha}, \infty\right]\right)$.

\section{An existence theorem of $\widehat{f}(s)$ in $H K(\mathbf{R}) \cap B V([ \pm \infty])$}

Let:

$$
\begin{gathered}
H K_{l o c}(\mathbf{R})=\{f: \mathbf{R} \rightarrow \mathbf{R} \mid f \in H K(I), \text { for any compact interval } I \subset \mathbf{R}\} \\
B V_{0}([ \pm \infty])=\{f: \mathbf{R} \rightarrow \mathbf{R} \mid f \in B V([a, \infty]) \cap B V([-\infty, b]), \text { for some } \\
\left.\qquad a, b \in \mathbf{R}, y \lim _{|x| \rightarrow \infty} f(x)=0\right\} \\
B V([ \pm \infty])=\{f: \mathbf{R} \rightarrow \mathbf{R} \mid f \in B V([a, \infty]) \cap B V([-\infty, b]), \\
\quad \text { for some } a, b \in \mathbf{R}\} \text { y }
\end{gathered}
$$

$\widehat{f}(s)=\int_{-\infty}^{\infty} e^{-i x s} f(x) d x$ the Fourier Transform of $f$ en $s, s \in \mathbf{R}$.

A part from [6, Proposition $\mathbf{2}$ b)] says that: If $f \in H K_{l o c}(\mathbf{R}) \cap$ $B V_{0}([ \pm \infty])$, then $\widehat{f}(s)$ exists for all $s \in \mathbf{R}$. As mentioned in the introductory section, with these conditions, it is not necessarily true the existence of $\widehat{f}(0)$. If $s \neq 0$, then the result is true.

For finding existence conditions of $\widehat{f}(s)$ for all $s \in \mathbf{R}$, the main idea is considering a proper subspace of $H K_{l o c}(\mathbf{R}) \cap B V_{0}([ \pm \infty])$. This subspace should be in such a way that it is not contained or it equals $L(\mathbf{R})$, because in this last way we will not have any advance. On the other hand, in order 
to have the existence of $\widehat{f}(0)$ we need that $f \in H K(\mathbf{R}$. The above sentence leads to us to think that the subspace might be

$$
H K(\mathbf{R}) \cap B V([ \pm \infty]) .
$$

Seeing that: $B V(\mathbf{R}) \subset B V([ \pm \infty])$ and that $\lim _{|x| \rightarrow \infty} f(x)=0$ if $f \in$ $H K(\mathbf{R}) \cap B V(\mathbf{R})$ or $f \in H K(\mathbf{R}) \cap B V([ \pm \infty])$, we have:

$$
\begin{aligned}
& H K(\mathbf{R}) \cap B V(\mathbf{R}) \subset H K(\mathbf{R}) \cap B V([ \pm \infty]) \\
& \subset H K_{l o c}(\mathbf{R}) \cap B V_{0}([ \pm \infty]) .
\end{aligned}
$$

Taking into account (3.1) and the result from section 2, then the following theorem is true for a larger space.

Theorem 3.1. If $f \in H K(\mathbf{R}) \cap B V([ \pm \infty])$, then $\widehat{f}(s)$ exists for all $s \in \mathbf{R}$,

Proof. If $s \neq 0$, we follow the proof from [5] and [6]. The result is true for $s=0$ because $f \in H K(\mathbf{R})$.

\section{References}

[1] Bartle, R.G., A Modern Theory of Integration, Graduate Studies in Mathematics, Vol 32, American Mathematical Society, Providence Rhode Island, (2001).

[2] Gordon, R. A., The Integral of Lebesgue, Denjoy, Perron, and Henstock, Graduate Studies in Mathematics, Vol 4, American Mathematical Society, Providence, (1994).

[3] Henstock, R., Lectures on The Theory of Integration, World Scientific Publications Co., Singapure, (1988).

[4] Lee Peng, Y., Lanzhou Lectures on Henstock Integration, Publications Co., Singapure, (1989).

[5] Y. E. Morales Rosado y F. J. Mendoza Torres, Algunos Aspectos de la Transformada de Fourier en el Espacio de las Funciones HK-Integrables, Aportaciones Matemáticas, Memorias SMM, 37, pp. 101-114, (2007). 
[6] Talvila Erik, Henstock-Kurzweil Fourier Transforms, Illinois Journal of Mathematics, 46, pp. 1207-1226, (2002).

Francisco Javier Mendoza Torres

Facultad de Ciencias Físico Matemáticas

Benemérita Universidad Autónoma de Puebla

Puebla, Pue., México, 72570

México

e-mail : jmendoza@fcfm.buap.mx

Juan Alberto Escamilla Reyna

Facultad de Ciencias Físico Matemáticas

Benemérita Universidad Autónoma de Puebla

Puebla, Pue., México, 72570

México

e-mail : jescami@fcfm.buap.mx

and

Ma. Guadalupe Raggi Cárdenas

Facultad de Ciencias Físico Matemáticas

Benemérita Universidad Autónoma de Puebla

Puebla, Pue., México, 72570

México

e-mail : gperaggi@fcfm.buap.mx 